\title{
Efectos de la intervención en conciencia morfológica sobre la lectura, escritura y comprensión: Meta-análisis
}

\author{
Mercedes I. Rueda-Sánchez * y Patricia López-Bastida
}

Dpto. de Ps. Evolutiva y de la Educación. Facultad de Psicología. Universidad de Salamanca (España).

\begin{abstract}
Resumen: El objetivo es comprobar, mediante meta-análisis, el impacto de las intervenciones en conciencia morfológica sobre, la lectura, escritura, comprensión y vocabulario de los escolares. Se han incluido en el metaanálisis 31 estudios, obtenidos de 19 artículos que cumplen los criterios de selección. La instrucción en conciencia morfológica tiene un tamaño del efecto medio alto y significativo en las variables de alfabetización estudiadas. En lectura, $g=0.473, S E=0.096, C I=0.284$ a $0.662, p=.000$, escritura, $g=$ $0.491, S E=0.078, C I=0.339$ a $0.643, p=.000$, comprensión, $g=0.468$, $S E=0.123, C I=0.227$ a $0.708, p=.000$ y vocabulario, $g=0.501, S E=0.152$, $C I=0.203$ a $0.798, p=.001$. El test de heterogeneidad Q resulta significativo sólo en escritura, por lo que fueron exploradas otras variables moderadoras, no encontrando diferencias entre los grupos. Se demuestra que el entrenamiento en conciencia morfológica tiene un impacto importante en la lectura, vocabulario y comprensión de los escolares sin y con dificultades lectoras. Mientras que en escritura los resultados son más heterogéneos.

Palabras clave: Meta-análisis; conciencia morfológica; lectura; escritura; vocabulario, dificultades; entrenamiento.
\end{abstract}

Title: Effects of morphological awareness training on reading, writing and comprehension: Meta-analysis.

Abstract: The aim is to check, through a meta-analysis, the impact of morphological awareness training on writing, reading, comprehension and vocabulary of grade schooler. 31 studies were included in the meta-analysis; they were obtained from 19 articles that meet the selection criteria. Morphological awareness instruction has a high-medium and significant effect size in studied variables of literacy. On writing, $g=0.491, S E=0.078$, $I Q=0339-0643, p=.000$, reading, $g=0.473, S E=0.096, I Q=0284-0662$, $p=.000$, comprehension, $g=0.468, S E=0.123, I Q=0227-0708, p=.000$ and finally vocabulary, $g=0.501, S E=0.152, I Q=0203-0798, p=.001$. The test of Heterogeneity $Q$ is only significant on writing so other moderating variables were explored but no differences between groups were found. It shows morphological awareness training improves reading, vocabulary and comprehension of grade schooler with and without reading difficulties. Nevertheless, the results on writing are more heterogeneous.

Key words: Meta-analysis; morphological awareness; reading; writing; difficulties; vocabulary; training.

\section{Introducción}

El objetivo de este trabajo es conocer el impacto de la intervención en conciencia morfológica sobre el aprendizaje de la lectura, escritura, vocabulario y comprensión, en escolares de diferentes niveles y con y sin dificultades de aprendizaje lector.

Cada vez son más abundantes los estudios que refieren que la conciencia morfológica favorece el desarrollo de estas competencias lecto-escritoras (Ramírez, Chen, Geva y Kiefer, 2010) y, la falta de conciencia morfológica puede provocar dificultades en el lenguaje escrito (Carlisle, 1987). Dificultades que pueden persistir más allá de la educación primaria (Nagy, Berninger, Abbott, Vaughan, y Vermeulen, 2003). Cabe pensar que la intervención en esta habilidad metalingǘstica puede favorecer el desarrollo de la propia conciencia morfológica e influir en un mejor progreso en el lenguaje escrito (Casalis y Colé, 2009). Es en este aspecto de la intervención donde radica el interés que ha motivado este estudio. Nos hemos propuesto conocer cómo se está trabajando esa competencia metalingüística a partir de una instrucción explícita. Hemos querido revisar ¿qué se hace en la instrucción en conciencia morfológica?, ¿quién lo hace?, ¿cuándo se hace?, ¿a quién va dirigido?, ¿cómo se hace?, ¿qué resultados se obtienen? y ¿cómo repercute en la lectura y escritura de quienes son entrenados?

Para dar sentido y valor a nuestro objetivo debemos revisar y entender qué es la conciencia morfológica y qué sabemos de su relación con el aprendizaje del lenguaje escrito.

* Dirección para correspondencia [Correspondence address]:

Mercedes I. Rueda. Facultad de Psicología. Avda. de la Merced 109-131.

CP 37005 Salamanca (España). E-mail: mirueda@usal.es reflexionar sobre la formación de las palabras y sus reglas así como manipular las estructuras morfológicas que la componen (Kuo y Anderson, 2006). Esta conciencia se va desarrollando a medida que avanza el aprendizaje del lenguaje escrito en educación primaria e incluso secundaria (Rubin, 1991), de manera que no es fruto exclusivo de la maduración o la exposición a la lengua. De esta forma, podemos establecer una clara distinción entre morfología y conciencia morfológica, de manera que la morfología se adquiere con el desarrollo del lenguaje oral y la conciencia morfológica se adquiere con el aprendizaje de la lectoescritura. Es importante tener en cuenta que la conciencia morfológica está relacionada con el resto de habilidades metalingüísticas y con el buen aprendizaje de la lectura, la escritura y la comprensión lectora (Kieffer y Lesaux, 2012b). Casalis y Louis-Alexandre (2000) consideran que los análisis morfológicos que realizan los estudiantes en el último curso de infantil pueden predecir e influir en el nivel de lectura en los dos primeros cursos de primaria.

Los niños escolarizados en primaria no sólo son capaces de leer y reconocer la estructura morfológica de las palabras, sino que también pueden extraer el significado de palabras complejas a través del análisis morfológico de sus diferentes partes. El conocimiento de los morfemas influye en el reconocimiento de las palabras de tal manera que para leer y acceder al significado es importante reconocer los diferentes morfemas y posteriormente ensamblarlos (Domínguez, Cuetos y Segui, 2000). Se constata también, la contribución que tiene la conciencia morfológica en la comprensión lectora (Deacon y Kirby, 2004) sobre todo por la influencia de la comprensión de las palabras y la amplitud del vocabulario. Tanto es así, que existen evidencias de que los alumnos con
La conciencia morfológica es una habilidad que permite 
un rápido crecimiento en conciencia morfológica, también muestran un rápido crecimiento en vocabulario (García y González, 2006). Tong, Deacon, Kirby, Cain y Parrilla (2011) evaluaron a niños de $2^{\circ}$ y $4^{\circ}$ de primaria en tareas de comprensión, y, posteriormente, se les administró una prueba de conciencia morfológica que evaluaba tanto la morfología flexiva, como la derivativa y composicional. Los resultados reflejaron que los niños con puntuaciones bajas en conciencia morfológica eran los que presentaban dificultades en la comprensión lectora.

También la escritura de palabras depende, en alguna medida, de que los niños entiendan que las palabras están compuestas por una estructura morfo-fonémica, es decir, que los elementos sonoros se combinan para formar morfemas, bien sea de manera flexiva, derivativa o por composición (Rubin, 1991). Es a partir de $2^{\circ}$ y $3^{\circ}$ de primaria cuando el niño aumenta sus conocimientos sobre las flexiones y comienza a utilizar formas derivadas en su escritura espontánea (Carlisle, 1996). Es más, la conciencia morfológica influye y predice el aprendizaje de la escritura, pero también es este aprendizaje de la lectura y escritura el que permite que los niños obtengan gran parte de sus conocimientos acerca de los morfemas (Nunes, Bryant y Bindman, 2006). Carlisle (1996) seleccionó alumnos de $2^{\circ}$ y $3^{\circ}$ grado de primaria con y sin dificultades de aprendizaje y analizó la precisión en el uso de morfemas flexivos, derivativos y palabras compuestas en la escritura espontánea. Los resultados mostraron diferencias significativas por grado y grupo. Los alumnos de segundo grado con dificultades eran menos precisos que sus compañeros en el uso de la morfología, mientras que los alumnos de tercer grado con y sin dificultades tenían mayores niveles de precisión.

Tenemos argumentos suficientes en favor de la importancia de la conciencia morfológica en el aprendizaje y desarrollo de la lectura y escritura y de las diferencias entre los buenos y malos lectores en relación a la conciencia morfológica (Rueda e Incera, 2011) por lo que es de gran interés determinar el peso de la intervención.

Pretendemos por lo tanto, aportar un análisis relevante y sistemático que nos permita valorar el peso de la intervención en conciencia morfológica sobre el aprendizaje de la lectura, escritura, comprensión y vocabulario. Para ello hemos desarrollado el estudio en dos fases, en primer lugar realizamos una descripción exhaustiva de las características que presentan los programas instruccionales en conciencia morfológica, para en un segundo momento realizar un metaanálisis que nos permita establecer el tamaño del efecto que presentan los estudios previamente analizados en cada una de las variables establecidas: lectura de palabras, escritura de palabras, comprensión y vocabulario.

\section{Método}

\section{Selección de la muestra}

La selección de la muestra de se ha realizado a partir de búsquedas en PsycINFO, Google Scholar y Psicodoc. Los criterios de búsqueda y selección han sido: a) artículos científicos a texto completo escritos en lengua inglesa o castellana, entre 1989 y 2013, b) programas de instrucción en conciencia morfológica en cualquiera de sus niveles: flexión, derivación y composición, que hubiesen sido puestos en práctica, aunque formasen parte de un programa más amplio de entrenamiento en competencias metalingüísticas, c) dirigidos a alumnos escolarizados independientemente de la etapa educativa: infantil, primaria y/o secundaria, d) destinados a niños con o sin dificultades en el aprendizaje de la lectoescritura, e) estudios en los que se evalúan los efectos de la instrucción en conciencia morfológica sobre lectura, escritura, comprensión y/o vocabulario, f) estudios que proporcionan estadísticos suficientes para calcular el tamaño del efecto.

Las palabras claves utilizadas en la búsqueda fueron, morphology, en combinación con instruction, program, vocabulary, reading y spelling para inglés. Y morfología, conciencia morfológica, instrucción y programa para castellano.

Partimos de una muestra inicial de 100 artículos. De ellos 75 fueron excluidos por no ser estudios instruccionales, es decir, a pesar de tratar la temática de la instrucción en conciencia morfológica no planteaban ni desarrollaban un programa de entrenamiento específico. Nos quedamos con 25 artículos instruccionales que analizamos de manera sistemática y descriptiva en este trabajo. Al finalizar el análisis de los artículos detectamos que sólo 19 proporcionaban estudios con los estadísticos suficientes para calcular el tamaño del efecto, por lo que son los que incluimos en el meta-análisis.

\section{Codificación de estudios}

Los estudios han sido codificados a partir de cuatro categorías de análisis: 1) características generales de la instrucción, 2) características de la muestra, 3) características específicas del entrenamiento y 4) características metodológicas.

1) Características generales de la instrucción. Se ha codificado (a) idioma de origen, (b) instructor, (c) duración del programa de instrucción, codificado en $n^{\circ}$ de semanas de intervención, $\mathrm{n}^{\circ}$ de sesiones totales de entrenamiento multiplicado por los minutos que dura la sesión y, por último, se anota el tiempo dedicado exclusivamente al entrenamiento en conciencia morfológica. La variable (d) grupo de instrucción, indica si el entrenamiento es individual, en pequeño grupo (2a12 alumnos), en grupo grande o clase, y/o combinando las tres opciones. La (e) forma de entrenamiento puede ser combinado o exclusivo en conciencia morfológica.

2) Características de la muestra. Se codifica (a) tamaño de la muestra, (b) grado de escolarización de los sujetos, (c) edad de los participantes, (d) género, indicando si hay 
más niños o niñas $(>,<)$. Alumnos con (e) dificultades o sin dificultades y (f) criterios de selección de la muestra de alumnos con dificultades.

3) Características especificas del entrenamiento. Se categorizan tres variables: (a) tareas que deben realizar los sujetos entrenados, (b) contenido o tipo de morfología en el que se instruye, (c) formato o modo en el que se instruye, con tareas orales o lenguaje escrito.

4) Características metodológicas. Se han codificado: tipo de (a) diseño del estudio, (b) medidas de evaluación, (c) tipo análisis de datos.

La fiabilidad en la codificación, realizada por dos codificadores independientes, se calculó en base al porcentaje de acuerdo entre las variables codificadas antes y después de la puesta en común. El acuerdo varió, del $83 \%$ en la codificación de la variable: características generales de la muestra, al $100 \%$ en la elección de las publicaciones incluidas en el estudio final de meta-análisis. El índice Kappa de Cohen osciló entre 0.91-1.00. Las discrepancias se resolvieron por consenso antes del análisis de resultados.

\section{Resultados}

\section{Análisis descriptivo de los artículos con estudios instruccionales}

El análisis sistemático nos ha permitido comprender en profundidad las características de la instrucción en conciencia morfológica y tener a nuestro alcance un amplio número de variables que nos puedan ayudar a entender la posible variabilidad de los resultados que encontremos en el estudio de meta-análisis posterior.

El idioma de las propuestas de entrenamiento más habitual es el inglés en el $66 \%$ de los estudios, seguido por el chino $12 \%$, persa, francés $7 \%$, danés y noruego $4 \%$. El instructor o persona que realiza el entrenamiento, es en un 55\% de los estudios el profesor habitual del aula, en un $41 \%$ de los casos son los investigadores y sólo en el $4 \%$ lo realizan los padres en sus hogares. Cuando la instrucción es realizada por profesores y/o padres estos son previamente entrenados por los investigadores. Los datos de la duración de la instrucción se ofrecen en minutos en el $89 \%$ de los estudios, de ese porcentaje, a su vez, en el $48 \%$ de estudios se indica el tiempo dedicado al entrenamiento exclusivo en conciencia morfológica que oscila, entre 300' y 600' (en el 46\% de los casos) y entre los 600' y los 1000 ' en el $53 \%$ de esos estudios que lo especifican. La instrucción se realiza en pequeño grupo de 2 a 12 alumnos (en el $36 \%$ de los casos), en grupo grande o grupo-clase (en el $32 \%$ de los estudios) y de forma individual en un 25\% de los casos analizados. En el 4\% de los estudios se combina el tamaño individual y pequeño grupo, y en el pequeño porcentaje restante no aparece este dato. En el 33\% de los estudios se entrena la conciencia morfológica de forma exclusiva, en los casos restantes se hace de forma combinada con otras habilidades como ortografía, vocabulario, lectura, análisis contextual (véase detalladamente la Tabla 1).

$\mathrm{Al}$ revisar las características de las muestras que se utilizan en los estudios instruccionales (véase Tabla 2), observamos que el tamaño de las muestras es inferior a 50 alumnos en el 33\% de los estudios, entre 50 y en 200 en el 40.5\% y, superior a 200 en el $26 \%$ de los casos, siendo el tamaño máximo 805 niños en un único estudio.

La variable género es desconocida en el 33\% de los estudios. En las investigaciones en las que sí se identifica (en el $67 \%$ ), el $72 \%$ de la muestra está compuesta por más niños que niñas. La edad de los sujetos que participan en los estudios va de los 5 a los 15 años. Situándose el porcentaje más alto en el intervalo de edad entre los 8 y los 11 años.

En el $48 \%$ de los estudios participan alumnos que tienen dificultades de aprendizaje en la lectura. Todos los programas de instrucción que incluyen en su muestra alumnos con dificultades, añaden criterios de selección, y de ellos, el 90\% incluye más de un criterio. Estos criterios son generalmente tres: bajo rendimiento en pruebas de lecto-escritura $(60 \%)$, puntuación promedio en test que evalúan el $C I(60 \%)$ y, ausencia de déficits o discapacidades asociadas (25\%). Un 16\% de los estudios que incluyen criterios de selección, no recogen en su muestra alumnos con dificultades lectoras. En este caso los criterios son relativos al idioma en el que hablan los alumnos, los de habla nativa inglesa, francesa o portuguesa, hablantes de lenguas minoritarias y/o los que aprenden dichos idiomas como lengua extranjera. Si revisamos las características específicas del entrenamiento en conciencia morfológica (ver Tabla 3), constatamos que el contenido de la instrucción se centra, en el $67 \%$ de los estudios, en un único nivel de morfología, bien derivativa, flexiva o composición. En el 33\% restante se combinan diferentes niveles de entrenamiento en conciencia morfológica. 
Tabla 1. Codificación de las características generales de la instrucción.

\begin{tabular}{|c|c|c|c|c|c|}
\hline \multirow[b]{2}{*}{ ESTUDIO } & \multicolumn{5}{|c|}{ CARACTERÍSTICAS GENERALES DE LA INSTRUCCIÓN } \\
\hline & IDIOMA & INSTRUCTOR & DURACIÓN & $\begin{array}{l}\text { GRUPO } \\
\text { INSTRUC. }\end{array}$ & $\begin{array}{l}\text { COMBINA/ } \\
\text { EXCLUSIVO }\end{array}$ \\
\hline 1. Abbott y Berninger (1999) & Inglés & Investigadores & $\begin{array}{l}\text { 16semanas } / / 16 \text { sesiones } \\
60^{\prime}=960^{\prime} / / 16 \times 25^{\prime}=400^{\prime}\end{array}$ & ${ }^{\mathrm{x}}$ Individual & $\begin{array}{l}\mathrm{CM} / \mathrm{L}, \mathrm{CL}, \mathrm{CF}, \\
\mathrm{O}\end{array}$ \\
\hline 2. Arnbak y Elbro (2000) & Danés & $\begin{array}{l}\text { Profesor de apo } \\
\text { yo }\end{array}$ & ${ }^{-} 12$ semanas $/ / 36$ sesiones x $15^{\prime}=540^{\prime}$ & Pequeño grupo & Exclusivo CM \\
\hline 3. Baumann et al. (2002) & Inglés & Investigadores & 4 semanas $/ / 12$ sesiones $x$ 50'=600'//NA & Gran grupo & $\mathrm{CM} / \mathrm{AC}$ \\
\hline 4. Baumann et al. (2003) & Inglés & Profesor & $\mathrm{NA} / / 25$ sesiones $\times 30^{\prime}=750 / / 25 \times 15^{\prime}=375$ & Gran grupo & $\mathrm{CM} / \mathrm{AC}, \mathrm{V}$ \\
\hline 5. Berninger et al (2008) & Inglés & Profesor & $\begin{array}{l}14 \text { semanas } / / 14 \text { sesiones x } 120^{\prime}=1680^{\prime} / / 14 \mathrm{x} \\
60^{\prime}=840^{\prime}\end{array}$ & $\mathrm{x}_{\text {Pequeño grupo }}$ & CM/O,Ppio.A \\
\hline 6. Bowers y Kirby (2010) & Inglés & Profesor & 5 semanas $/ / 20$ sesiones $\times 50^{\prime}=1000^{\prime}$ & Gran grupo & Exclusivo CM \\
\hline 7. Carlo et al (2004) & Inglés & Profesor & 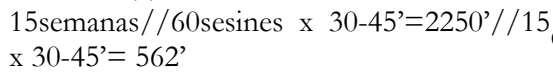 & 5 Gran grupo & $\mathrm{CM} / \mathrm{V}$ \\
\hline 8. Casails y Colé (2009) & Francés & Investigador & 9 semanas / /12sesiones x 30'=360'//NA & Pequeño grupo & $\mathrm{CM} / \mathrm{CF}$ \\
\hline 9. Chow et al (2008) & Chino & Padres & 12semanas $/ / 24$ sesiones x $20^{\prime}=480^{\prime} / / \mathrm{NA}$ & Individual & $\mathrm{CM} / \mathrm{L}$ \\
\hline 10. a. Hamed Ghaemi (2009) & Persa & Investigadores & 14 semanas $/ / 14$ sesiones x $30^{\prime}=420^{\prime} / / \mathrm{NA}$ & Pequeño grupo & $\mathrm{CM} / \mathrm{CF}$ \\
\hline b. Hamed Ghaemi (2009) & Persa & Investigadores & 22 semanas $/ / 22$ sesiones x 50' $=1100^{\prime} / / \mathrm{NA}$ & Individual & $\mathrm{CM} / \mathrm{CF}$ \\
\hline 11. Good (2011) & Inglés & Profesor & 10 semanas $/ / 20$ sesiones x 30' $=600^{\prime}$ & Pequeño grupo & Exclusivo CM \\
\hline 12. a.Griva y Anastasiou (2009) & Inglés & Profesor & 24 semanas $/ / 7$ sesiones $x$ 45' $=315^{\prime}$ & Gran grupo & Exclusivo CM \\
\hline b. Griva y Anastasiou (2009) & Inglés & Profesor & 24 semanas $/ / 7$ sesiones $\mathrm{x} 45^{\prime}=315^{\prime}$ & Pequeño grupo & Exclusivo $\mathrm{CM}$ \\
\hline 13. Henry (1989) & Inglés & Profesor & NA//25sesiones x 30-45'=937'//NA & $\mathrm{NA}$ & $\mathrm{CM} / \mathrm{CF}$ \\
\hline 14. Kieffer y Lesaux (2012a) & Inglés & Profesor & NA & Gran grupo & Exclusivo CM \\
\hline 15. Kirk y Gillon (2009) & Inglés & Investigador & 9.5 semanas $/ / 19.4$ sesiones x $45^{\prime}=873^{\prime} / / \mathrm{NA}$ & $\begin{array}{l}\text { Individual y pe- } \\
\text { queño grupo }\end{array}$ & $\mathrm{CM} / \mathrm{CF}, \mathrm{O}$ \\
\hline 16. Lovett y Steinbach (1997) & Inglés & Profesor & NA//35sesiones x 60'=2100'//NA & Pequeño grupo & $\mathrm{CM} / \mathrm{CF}$ \\
\hline 17. Lovett et. al (2000) & Inglés & Profesor & 14 semanas $/ / 70$ sesiones x $60^{\prime}=4200^{\prime} / / \mathrm{NA}$ & Individual & $\mathrm{CM} / \mathrm{CF}$ \\
\hline 18. Lyster (2002) & Noruego & $\begin{array}{l}\text { Profesor } \\
\text { ayuda }\end{array}$ & $\begin{array}{l}17 \text { semanas } / / 17 \text { sesiones } \\
40^{\prime}=595^{\prime} / / \text { NA } \\
17 \text { semanas } / / 34 \text { sesiones } \\
20^{\prime}=595^{\prime} / / \text { NA }\end{array}$ & Pequeño grupo & $\mathrm{CM} / \mathrm{CF}$ \\
\hline $\begin{array}{l}\text { 19. Nunes, Bryant y Olsson } \\
(2003)\end{array}$ & Inglés & Investigador & 12 semanas $/ / 12$ sesiones $x 30^{\prime}=360^{\prime} / / \mathrm{NA}$ & Pequeño grupo & $\mathrm{CM} / \mathrm{CF}$ \\
\hline 20. Packard et. al (2006) & Chino & Profesor & 10semanas//NA//NA & Gran grupo & $\mathrm{CM} / \mathrm{O}$ \\
\hline 21. Rosa y Nunes (2008) & Portugués & Investigador & $\mathrm{NA} / / \mathrm{NA} / / \mathrm{NA}$ & Gran grupo & Exclusivo CM \\
\hline 22. St-Pierre y Dubé (2012) & Francés & Investigador & 10semanas $/ / 25$ sesiones $\mathrm{x} 40^{\prime}=1000^{\prime}$ & Pequeño grupo & Exclusivo CM \\
\hline 23. Tsesmeli y Seymour (2009) & Inglés & Investigador & NA//16sesiones $\times$ 40’ $=640^{\prime}$ & Individual & Exclusivo CM \\
\hline 24. a. Vadasay et. al (2006) & Inglés & Profesor & $\begin{array}{l}\text { 20semanas } / / 80 \text { sesionesx } 30^{\prime}=2400 / / 40 \times 15^{\prime} \\
=600^{\prime}\end{array}$ & Individual & $\mathrm{CM} / \mathrm{CF}$ \\
\hline b. Vadasay et. al (2006) & Inglés & Profesor & $\begin{array}{l}\text { NA//72sesiones } \\
54 \times 15^{\prime}=810^{\prime}\end{array}$ & Individual & $\mathrm{CM} / \mathrm{CF}$ \\
\hline 25. Wu et. al (2009) & Chino & Profesor & $\begin{array}{l}\text { NA//2sesiones al día de 45'. Todo curso } \\
\text { escolar }\end{array}$ & Gran grupo & Exclusivo CM \\
\hline
\end{tabular}

NA(no hay datos), CM(conciencia morfológica), L(lectura), CL(comprensión), CF(conciencia fonológica), O(ortografía), AC(análisis contextual), Ppio.A(principio alfabético)

Tabla 2. Codificación de las características de la muestra.

CARACTERÍSTICAS DE LA MUESTRA

\begin{tabular}{|c|c|c|c|c|c|c|}
\hline \multirow[b]{2}{*}{$\begin{array}{l}\text { ESTUDIO N } \\
\text { Citados en la } \\
\text { Tabla } 1\end{array}$} & \multicolumn{6}{|c|}{ CARACTERISTICAS DE LA MUESTRA } \\
\hline & GRADO & $\begin{array}{l}\text { EDAD } \\
\text { (años) }\end{array}$ & TAMAÑO $(N)$ & GÉNERO & DIFICULTADES & $\begin{array}{l}\text { CRITERIOS DE } \\
\text { SELECCIÓN }\end{array}$ \\
\hline 1. & $4^{\circ}, 5^{\circ}, 6^{\circ}, 7^{\circ}$ & $9 \mathrm{a} 13$ & $20(10 \mathrm{CM}+10 \mathrm{GC})$ & $\begin{array}{l}13 \text { niños } \\
7 \text { niñas }\end{array}$ & Sí & $\begin{array}{l}\text { - Bajo rendimiento lector } \\
\text { - Promedio CI verbal } \\
\text { - Bajo rendimiento lector }\end{array}$ \\
\hline 2. & $4^{\circ}, 5^{\circ}$ & $10 \mathrm{a} 12$ & $60(33 \mathrm{CM}+27 \mathrm{GC})$ & $\begin{array}{l}40 \text { niños } \\
20 \text { niñas }\end{array}$ & $\begin{array}{l}\text { Sí } \\
\text { Disléxicos }\end{array}$ & $\begin{array}{l}\text { - Promedio lenguaje oral y } \\
\text { HH.Cognitivas } \\
\text { - Reciben clases apoyo }\end{array}$ \\
\hline 3. & $5^{\circ}$ & $10-11$ & $88(24 \mathrm{CM}+21 \mathrm{CMyCTXTO+21GC)}$ & $N A$ & No & NA \\
\hline 4. & $5^{\circ}$ & $10-11$ & 157 (78CM+79GC) & $N A$ & No & NA \\
\hline
\end{tabular}




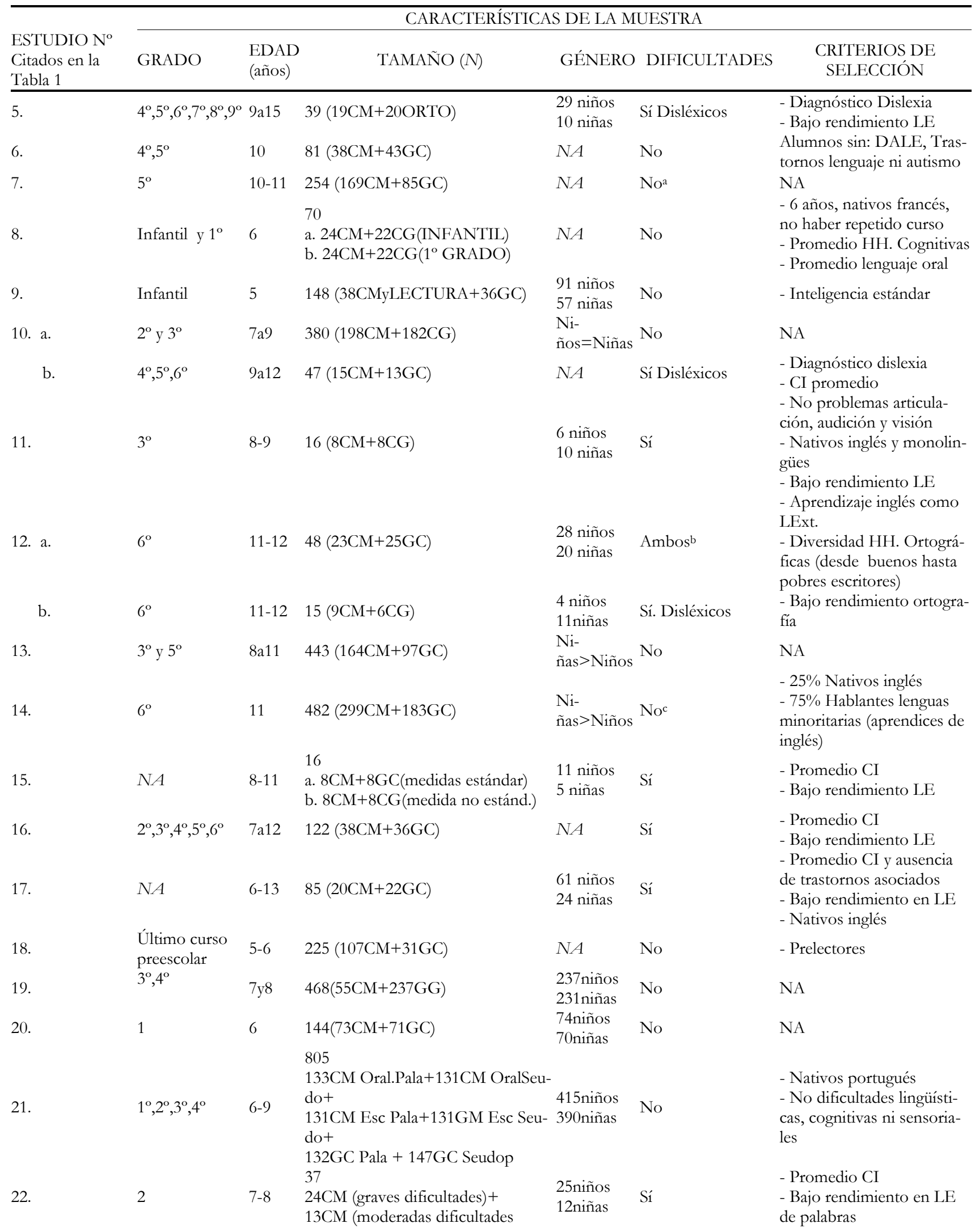




\begin{tabular}{|c|c|c|c|c|c|c|}
\hline \multirow[b]{2}{*}{$\begin{array}{l}\text { ESTUDIO No } \\
\text { Citados en la } \\
\text { Tabla } 1\end{array}$} & \multicolumn{6}{|c|}{ CARACTERÍSTICAS DE LA MUESTRA } \\
\hline & GRADO & $\begin{array}{l}\text { EDAL } \\
\text { (años) }\end{array}$ & TAMAÑO $(N)$ & GÉNERO & DIFICULTADES & $\begin{array}{l}\text { CRITERIOS DE } \\
\text { SELECCIÓN }\end{array}$ \\
\hline 23. & $\begin{array}{l}4^{\circ}, 5^{\circ}, 6^{\circ}, 7^{\circ} y \\
1^{\circ}, 2^{\circ}, 3^{\circ} \\
\text { Secundaria }\end{array}$ & $8 \mathrm{a} 14$ & $\begin{array}{l}68 \\
9 \mathrm{CM}+14 \mathrm{GC}(=\mathrm{edad})+22 \mathrm{GC}(=\text { nivel } \\
\text { ortografía })+23 \mathrm{GC}(=\text { nivel lectura })\end{array}$ & $N A$ & Ambos $^{d}$ & $\begin{array}{l}\text { GE:Bajo rendimiento LE y } \\
\text { ausencia déficits asociados } \\
\text { GC: Promedio LE }\end{array}$ \\
\hline 24. a. & $2^{\circ}, 3^{\circ}$ & 8 & $31(12 \mathrm{CM}+19 \mathrm{GC})$ & $\begin{array}{l}\mathrm{Ni}- \\
\text { ños }>\text { Niñas }\end{array}$ & Sí & $\begin{array}{l}\text { - No repetir } \\
\text { - No apoyo } \\
\text { - Bajo rendimiento } \mathrm{L}\end{array}$ \\
\hline b. & $2^{\circ}, 3^{\circ}$ & 8 & $21(11 \mathrm{CM}+10 \mathrm{CG})$ & $\begin{array}{l}\text { Ni- } \\
\text { ños }>\text { Niñas }\end{array}$ & Sí & $\begin{array}{l}\text { - No repetir } \\
\text { - No apoyo } \\
\text { - Bajo rendimiento } \mathrm{L}\end{array}$ \\
\hline 25. & $1^{\circ}, 2^{\circ}$ & 6 & $154(74 \mathrm{CM}+80 \mathrm{CG})$ & $\begin{array}{l}\text { 73niños } \\
\text { 73niñas }\end{array}$ & No & NA \\
\hline
\end{tabular}

a En el estudio 7, la muestra está compuesta por alumnos nativos de habla inglesa y por aprendices de inglés como lengua extranjera. GE(grupo entrenamiento) GC(grupo control)

${ }^{\mathrm{b}}$ En el estudio 12, los alumnos son entrenados y evaluados en inglés. Estos alumnos son aprendices de inglés como lengua extranjera, de forma que su idioma nativo es otro. (Promedio=puntuación media, $\mathrm{HH}=$ habilidades, $\mathrm{LExt}=$ lengua extranjera, $\mathrm{LE}=\mathrm{lec}$ )

c En el estudio 14, la muestra está compuesta por alumnos nativos de habla inglesa y por estudiantes de lenguas minoritarias que dominan el inglés.

d En el estudio 23, la muestra está formada por tres grupos. Un grupo lo componen alumnos de secundaria con dislexia. Otro grupo está compuesto por alumnos de secundaria sin dificultades con el mismo nivel en lectoescritura que los alumnos disléxicos. Y un tercer grupo está formado por alumnos de primaria sin dificultades pero con el mismo nivel de rendimiento que los alumnos disléxicos.

Tabla 3. Características específicas del entrenamiento en conciencia morfológica.

EST. TAREAS TORMA

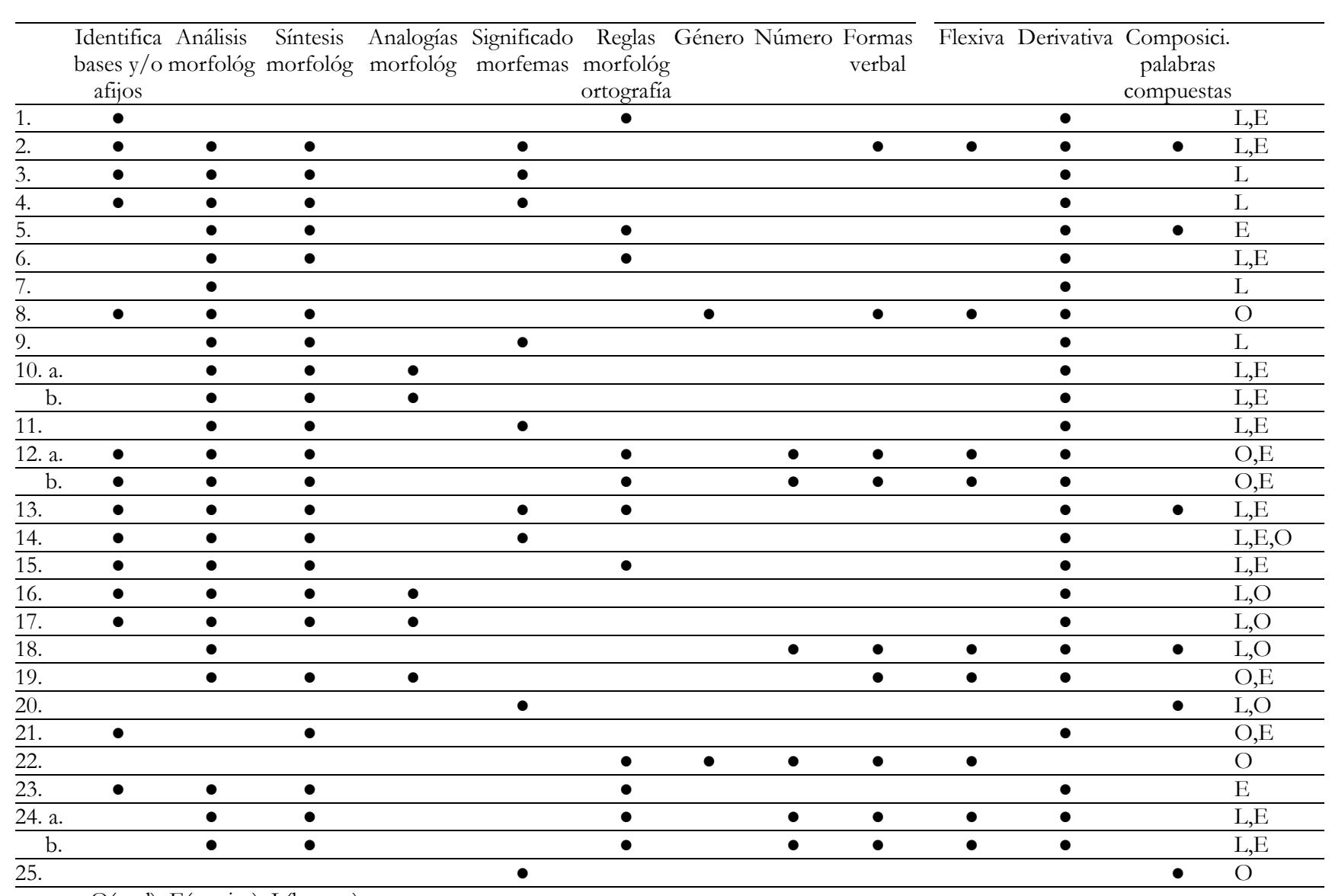

$\mathrm{O}$ (oral), E(escrito), L(lectura), 
Tabla 4. Codificación de las características metodológicas.

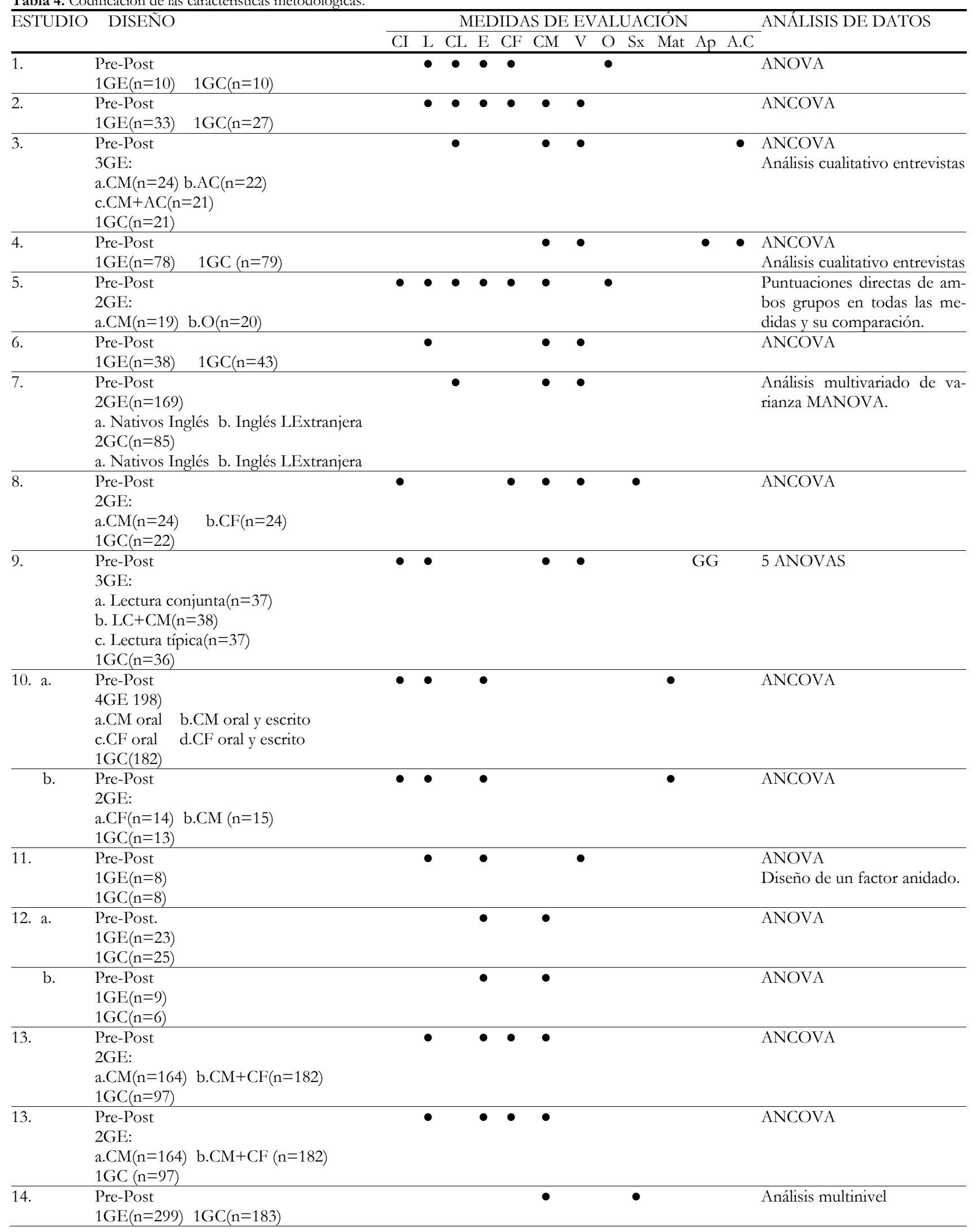




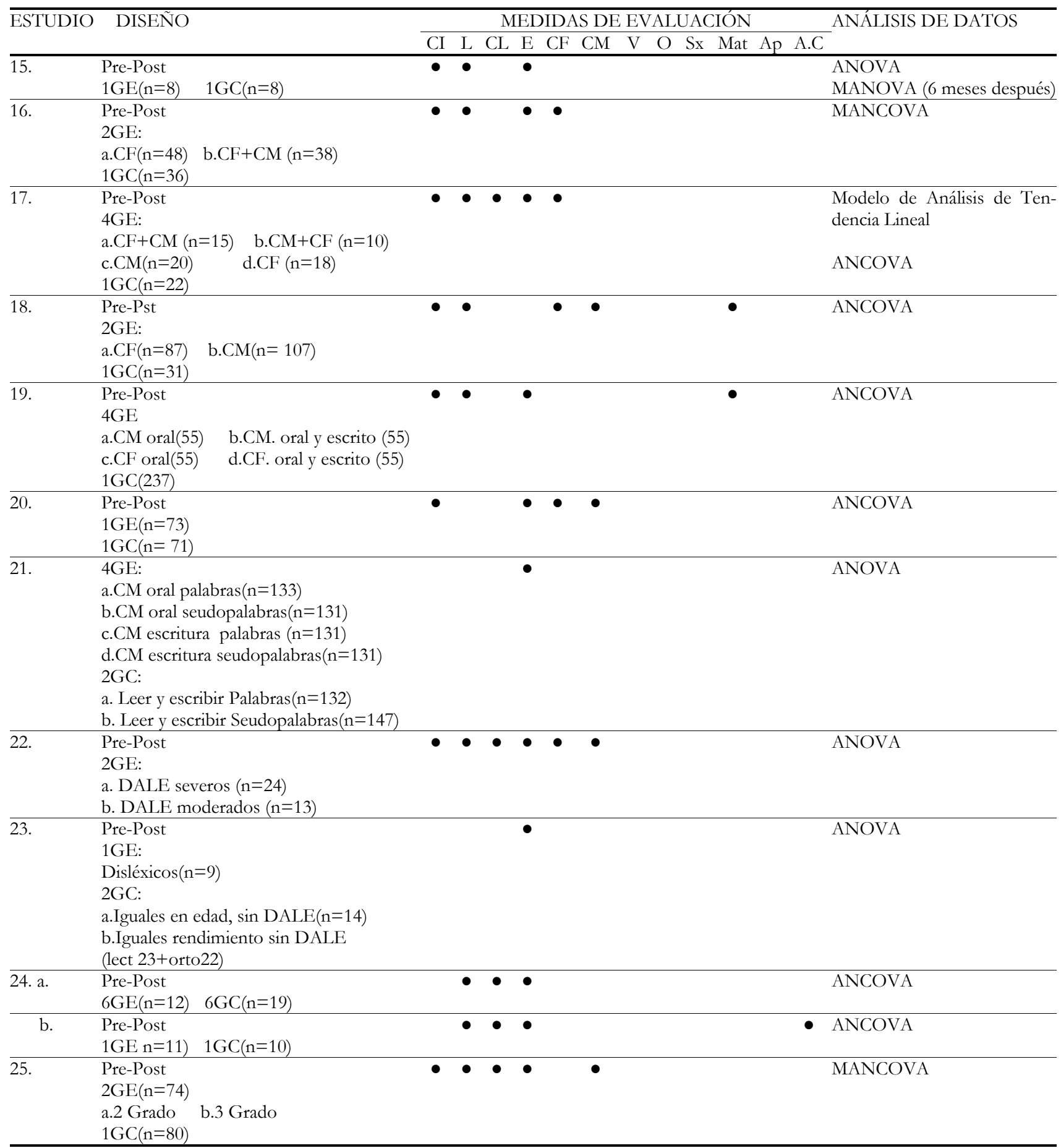

CI(cociente intelectual), L(lectura), CL(comprensión lectora), E(escritura), CF(conciencia fonológica), CM(conciencia morfológica), $\mathrm{V}$ (vocabulario, $\mathrm{O}$ (ortografía), Sx(análisis sintáctico), Mat(matemáticas), Ap(aprendizaje de contenidos, y AC(análisis contextual).

El tipo de tareas que se realizan en los niveles de morfología derivativa son fundamentalmente análisis $(28 \%)$, síntesis $(26 \%)$ e identificación de morfemas $(16 \%)$. En menor porcentaje, reglas $(12.5 \%)$, significado $(11 \%)$ y analogías $(6 \%)$.
En morfología flexiva se trabaja, género (13\%), número $(33 \%)$ y fundamentalmente formas verbales $(53 \%)$. Las tareas de composición se limitan a identificar y segmentar las palabras compuestas.

El formato en el que se realiza el entrenamiento en dichas tareas es oral combinado con tareas de lectura y/o escritura 
en el $63 \%$ de los estudios, frente al $37 \%$ donde la instrucción es exclusiva en un solo formato (oral $40 \%$, lectura $40 \%$, escritura 20\% ).

Por último, hemos revisado y analizado las características metodológicas que tienen los estudios instruccionales (véase Tabla 4). El tipo de diseño que predomina en los estudios analizados es un pretest-postest (en el 96\% de los casos), el resto son estudios con evaluación pos-test. Hay, por otro lado, gran variedad en cuanto al número y combinación de los grupos experimentales y grupos de control.

Encontramos diversidad, también, respecto a la forma de evaluación de los resultados de los sujetos, ocupando la escritura/ortografía el mayor porcentaje $(19 \%)$, le siguen pruebas de lectura $(17 \%)$, conciencia morfológica (13\%), fonológica $(10 \%)$, comprensión lectora $(10 \%)$ y vocabulario $(8 \%)$. El resto del porcentaje lo ocupan otro tipo de variables ajenas a la lectura y escritura.

Los procedimientos de análisis más frecuentes son el Análisis de Co-Varianza (45\%) y el Análisis de Varianza $(25 \%)$.

En el 89\% de los estudios se confirman mejoras en el rendimiento de diferentes habilidades, especialmente en escritura de palabras y seudopalabras $(56 \%)$, seguido por lectura de palabras y frases (41\%), comprensión lectora $(22 \%)$, adquisición de vocabulario (15\%) y conciencia fonológica $(15 \%)$. Además, en el $11 \%$ de los estudios se confirma una transferencia de dominio entre el rendimiento en conciencia morfológica y conciencia fonológica. En el 11\% de los estudios no hay mejoras significativas tras la intervención.

Respecto a los alumnos que obtienen mejoras en el rendimiento de las diferentes habilidades mencionadas, solo el $7 \%$ de los estudios afirman que los alumnos de la etapa de infantil, (5 y 6 años) pueden ser instruidos en conciencia morfológica y obtener beneficios de dicho entrenamiento. Frente al 93\% donde se defiende que las mejoras las obtienen los alumnos de primaria e incluso los escolarizados en los primeros años de secundaria. Del $48 \%$ de estudios que incluyen en su muestra alumnos con dificultades lectoras, en el $69 \%$ de los casos se afirma que estos niños obtienen mejoras en el rendimiento del proceso lecto-escritor.

\section{Meta-análisis. Cálculo del tamaño del efecto}

En el meta-análisis se incluyeron 19 de los 25 artículos previamente analizados y descritos. Todos aquellos que in- cluían los estadísticos necesarios para estimar el tamaño del efecto. De estos 19 artículos obtuvimos 31 estudios, debido a que en varios artículos encontramos que, o aparecen dos estudios bien diferenciados o, en un mismo estudio se subdividen los grupos y se ofrecen resultados diferentes procedentes de muestras distintas, bien por ser de curso escolar distinto, por las características de los sujetos, con/sin dificultades o, porque aplican la instrucción de forma diferente, etc.

El número total de sujetos que participan en estos estudios es de 2769 alumnos distribuidos entre grupo de entrenamiento (1518) y grupo de control (1251). Hemos calculado el tamaño del efecto y realizado el meta-análisis en lectura (Grupo Entrenamiento GE=362, Grupo Control GC=470), escritura ( $\mathrm{GE}=1394, \mathrm{GC}=1128)$, comprensión $(\mathrm{GE}=127$, $\mathrm{GC}=141)$ y vocabulario $(\mathrm{GE}=206, \mathrm{GC}=211)$.

Para la realización de todos los análisis empleamos la aplicación Comprehensive Meta-Analysis (CMA, v.2.06; Borenstein, Hedges, Higgins y Rothstein, 2009). Seleccionamos un modelo de efectos aleatorios dada la alta variabilidad observada entre los estudios (Raudenbush, 2009) y, como índice del tamaño del efecto de las diferencias entre el grupo de tratamiento y grupo de control, la $g$ de Hedges y Olkin (1985) que proporciona una estimación más conservadora que la $d$ de Cohen cuando se estima que un porcentaje de las muestras no son muy grandes, como puede ser en nuestro caso. Para la realización de los cálculos hemos utilizado las medias, desviaciones típicas y tamaño de los grupos. Cuando no se encontraron datos de medias y desviaciones típicas en los estudios, hemos utilizado la $F$ o la $t$.

Se utilizó la prueba $Q$ para evaluar la heterogeneidad de los tamaños del efecto (Cochran, 1977) junto con el índice I ${ }^{2}$ (Higgins, 2002; Huedo-Medina, Sánchez-Meca, MarínMartínez y Botella, 2006). Un valor significativo en el índice $Q$ sugiere que la distribución de tamaños del efecto alrededor de la media es mayor de lo que sería esperable sólo a partir de un error de muestreo. El estadístico $I^{2}$ cuantifica el grado de heterogeneidad estimando el porcentaje de la varianza que es atribuible a la variabilidad entre los estudios.

En la Tabla 5 se exponen los datos relacionados con el tamaño el efecto y los índices de heterogeneidad para cada una de las variables analizadas.

Tabla 5. Tamaño del efecto de la instrucción en conciencia morfológica sobre la lectura, escritura comprensión y vocabulario e índices de heterogeneidad.

\begin{tabular}{|c|c|c|c|c|c|c|c|c|c|c|}
\hline & \multicolumn{4}{|c|}{ Tamaño efecto } & \multicolumn{2}{|c|}{ I.C. al 95\% } & \multirow[b]{2}{*}{$Z$} & \multicolumn{3}{|c|}{ Heterogeneidad } \\
\hline & $k$ & $g$ & SE & $S^{2}$ & $L i$ & $L s$ & & $Q$ & $p$ & $I^{2}$ \\
\hline Lectura & 14 & 0.473 & 0.096 & 0.009 & 0.284 & 0.662 & $4.902^{*}$ & 18.758 & 0.131 & 30.695 \\
\hline Escritura & 26 & 0.491 & 0.078 & 0.006 & 0.339 & 0.643 & $6.323^{*}$ & 69.778 & 0.000 & 64.172 \\
\hline Vocabulario & 6 & 0.501 & 0.152 & 0.023 & 0.203 & 0.798 & $3.295^{*}$ & 10.240 & 0.069 & 51.170 \\
\hline Comprensión & 5 & 0.468 & 0.123 & 0.015 & 0.227 & 0.708 & $3.813^{*}$ & 1.913 & 0.752 & 0.000 \\
\hline
\end{tabular}

k-número de estudios, $g$-tamaño del efecto medio; SE-error_estándar; $S^{2}$-varianza: Li-Ls límites inferior-superior del intervalo de confianza al 95\%;

$Z$-prueba de hipótesis nula; $Q$-prueba de heterogeneidad; $I^{2}=$ varianza explicada.

$*=p<.05$ 
Los tamaños del efecto encontrados en los estudios que instruyen en conciencia morfológica y que miden su impacto en la lectura, comprensión y vocabulario de los escolares, podemos considerarlos, de acuerdo con Cohen (1988), como de intensidad media y homogéneos, es decir, que representan bien a los estudios (véase Tabla 5). Es interesarse resaltar que en los resultados obtenidos en comprensión, que aunque con una muestra pequeña $(k=5)$ el tamaño del efecto es importante $(g=0.468)$ y la homogeneidad alta $(Q=1.913, p=$ $\left..752, I^{2}=0.000\right)$. Vocabulario y lectura también muestran un buen tamaño del efecto y homogeneidad, pero la variabilidad en un $51.7 \%$ y $30.6 \%$ respectivamente tiene su origen en la heterogeneidad entre los estudios.
Con respecto a la escritura observamos que el tamaño del efecto es también de intensidad media y significativa. Sin embargo, los estadísticas de heterogeneidad para el modelo de efectos aleatorios confirmaron que su distribución es heterogénea con un valor $Q$ elevado y significativo $(Q=69.778$, $p=.000)$. La mayor parte de esta heterogeneidad, un $I^{2}=64.1 \%$ tiene su origen en la variabilidad entre los estudios más que en errores de las muestras lo que justifica la realización de análisis de moderadores.

Las tablas (1 al 4) proporcionan una visión general de las características de la muestra de estudios y de las posibles variables moderadoras: dificultad SI/NO, duración de la intervención, nivel escolar, entrenador, lengua de origen del estudio, forma de instrucción, etc.

Tabla 6. Análisis de las variables moderadoras en escritura.

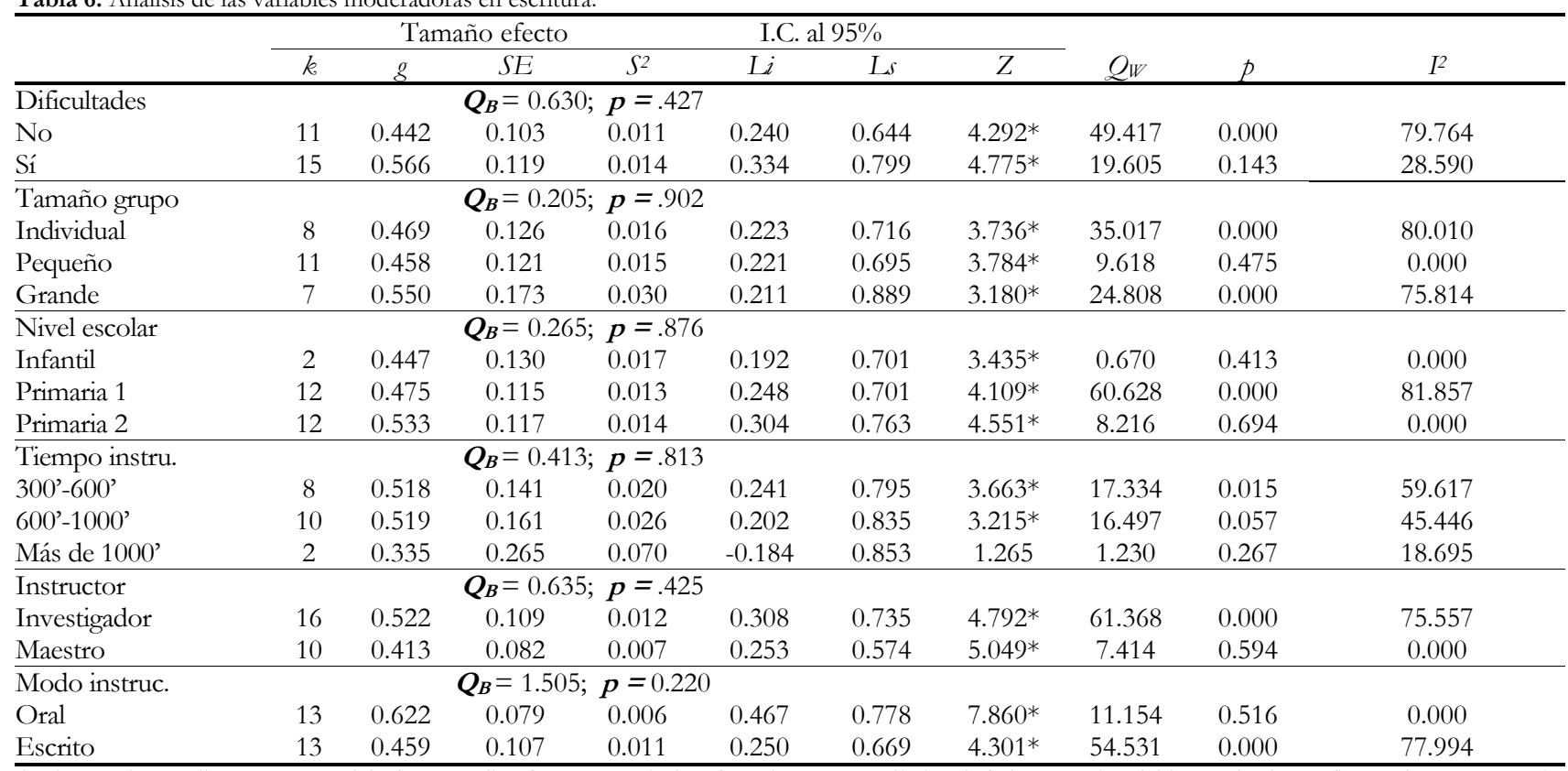

k-número de estudios; g-tamaño del efecto medio; SE-error estándar; $S^{2}$-varianza: Li-Ls límites inferior-superior del intervalo de confianza al $95 \%$; $Z$-prueba

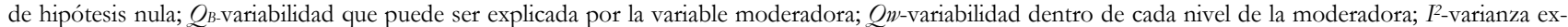
plicada.

$*=p<.05$.

Para el análisis de las variables moderadoras se aplicaron ANOVAs todos ellos asumiendo un modelo de efectos mixtos. Los resultados de los análisis, véase la Tabla 6 , no produjeron diferencias significativas entre los tamaños del efecto del tratamiento entre los niveles de cada una de las variables moderadoras establecidas como, por ejemplo, en relación con el nivel de dificultad de los sujetos $\left(Q_{B}=0.630\right.$, $p=.427)$. Sin embargo, si se constatan diferencias en el tamaño medio del efecto dentro de los distintos niveles de las variables moderadoras. Observamos que la variable dificultades presenta un tamaño del efecto mayor, significativo y homogéneo cuando los niños tienen dificultades que cuando no las tienen. Lo que nos permite interpretar con cierta seguridad que la instrucción en conciencia morfológica en los niños con dificultades tiene un buen impacto en su escritura.
La instrucción en grupo pequeño, frente a la instrucción en grupo grande o individual, ofrece también mejores índices (véase la Tabla 6). Es interesante valorar igualmente el tamaño del efecto para la variable instructor, siendo el maestro frente al investigador quien presenta un mayor índice de homogeneidad y lo mismo ocurre con la homogeneidad del tamaño del efecto de la instrucción con los alumnos de primaria $\left(1^{\circ}\right.$ a $\left.4^{\circ}\right)$ o en el modo de instrucción, en el que la instrucción que incluye alguna forma escrita, lectura o escritura, muestra mejores índices que en la que se emplea solo la forma oral. Por último, señalemos que no se encontraron diferencias en la homogeneidad del tamaño del efecto en la variable tiempo de instrucción.

El sesgo de publicación se analizó mediante la inspección visual de los gráficos funnel plot en los que no se evidenció 
asimetría. Las pruebas de regresión de Egger no fueron estadísticamente significativas (lectura: $\mathrm{t}=0.923, p=.374$; escritura: $t=0.281, p=.780$; comprensión: $t=3.008, p=.06$; vocabulario: $t=0.303, p=.777$ ) lo que permite descartar el sesgo de publicación como una amenaza contra la validez de los resultados.

\section{Conclusiones}

El propósito de este trabajo ha sido proporcionar una síntesis cuantitativa de la relevancia que tiene la instrucción en conciencia morfológica para provocar cambios sustanciales en la lectura y escritura de palabras, la comprensión y el vocabulario de los estudiantes con y sin dificultades de aprendizaje.

Los resultados que encontramos muestran unos tamaños de los efectos importantes, que ofrecen robustez a los estudios instruccionales en conciencia morfológica y que nos hacen plantear que la instrucción en conciencia morfológica tiene que considerarse una herramienta sustancial en el desarrollo de la alfabetización de los escolares desde finales de la etapa infantil (Lyster, 2002), y, sobre todo, en toda la etapa de primaria, llegando incluso a los primeros cursos de secundaría (Tsesmeli y Seymour, 2009). Los resultados apoyan el uso de la instrucción en conciencia morfológica en el aula, especialmente para los estudiantes que tienen dificultades con la lecto-escritura (McCutchen, Stull, Herrera, Lotas y Evans, 2014).

Hemos comprobado, asimismo, que la conciencia morfológica es un indicador clave en el desarrollo del vocabulario y comprensión lectora (Park, 2015). Trabajar con los elementos morfológicos de las palabras permite a los alumnos tomar conciencia de su significado más profundo, les ayuda a reconocer partes familiares en palabras menos familiares, repercutiendo en el progreso y adquisición del vocabulario. El vocabulario, a su vez, es una variable mediadora en la comprensión lectora. No obstante, parece interesante la idea de que la conciencia morfológica pueda ejercer una influencia directa sobre la comprensión, tal como algunos investigadores apuntan (Deacon, Kieffer y Laroche, 2014). Aun no está muy claro el mecanismo por el cual la concien-

\section{Referencias}

*Abbott, S. y Berninger, V. (1999). It's never too late remediate teaching word recognition to students with reading disabilities in Grade 4-7. Annals of Dyslexia, $49(1), 221-250$.

Arnbak, E. y Elbro, C. (2000). The effects of morphological awareness training on the reading and spelling skills of Young dyslexics. Scandinavian Journal of Educational Research, 44(3), 229-250.

Baumann, J. F., Carr Edwards, E., Boland, E.M., Olejnik, S. y Kame'enui, E.J. (2003). Vocabulary tricks: effects of instruction in morphology and context on fifth-grade students' ability to derive and infer word meanings. American Educational Research Journal, 40(2), 447-494.

Baumann, J. F., Carr Edwards, E., Font, G., Tereshinski, C.A., Kame'enui, E.J. y Olejnik, S. (2002). Teaching morphemic and contextual analysis to fifth-grade students. Reading Research Quarterly, 37(2), 150-176. cia morfológica influye sobre la comprensión, pero los datos nos inducen a pensar que es así. Perfetti (2007) defiende en esta línea, que las habilidades morfológicas contribuyen a la elaboración de representaciones léxicas de mayor calidad, lo que implica una más rápida recuperación del significado del texto. Comprender el mecanismo subyacente a la relación entre conciencia morfológica y comprensión sigue siendo un reto de investigación en la actualidad.

En la misma línea, los resultados tienen consecuencias para la práctica educativa. De tal manera que podemos plantear que la propuesta de instrucción, en conciencia morfológica, con un mayor impacto será aquella en que el objetivo es mejorar lectura de palabras, comprensión y vocabulario. Si el objetivo es mejorar la escritura de palabras, tendremos en cuenta que los resultados más potentes son los obtenidos con alumnos con dificultades lectoescritura en la etapa de primaria $\left(1^{\circ}\right.$ a $\left.4^{\circ}\right)$. La instrucción, ha de realizarse en grupos pequeños (2, 12 alumnos). Los entrenamientos son más efectivos si se vinculan a la dinámica general del aula y son los propios tutores de los niños quienes, previamente entrenados, desarrollan la instrucción. El objetivo ha de ser tomar conciencia de los tres niveles de morfología, flexiva, derivativa y composición. Para trabajar la morfología derivativa, se puede utilizar el análisis de la palabra de manera sustancial, pero también la síntesis e identificación de morfemas juegan un papel importante. Es interesante trabajar el uso de las reglas ortográficas, los significados de los morfemas y las analogías, para comprender los significados de las palabras y mejorar el vocabulario. La morfología flexiva puede trabajarse analizando género, número $\mathrm{y}$, fundamentalmente, las formas verbales.

Las limitaciones de este estudio son, a priori, las propias de todos los meta-análisis, como señalan Borenstein et al. (2009). No obstante, para investigaciones futuras una propuesta de mejora es focalizar la búsqueda en estudios instruccionales de conciencia morfológica que mida su repercusión sobre comprensión. A pesar de que hemos encontrado un buen tamaño del efecto en esta variable y no hemos constatado sesgo de publicación sí habría que asegurar este resultado con un mayor tamaño de la muestra.

Berninger, V.M., Winn, W.D., Stock, P., Abbott, R.D., Eschen, K., Lin, S-J., Garcia, N., Anderson-Youngstrom, M., Murphy, H., Lovitt, D., Trivedi, P., Jones, J., Amtmann, D., Nagy, W. (2008). Tier 3 specialized writing instruction for students with dyslexia. Reading and Writing: An Interdisciplinary Journal, 21(1-2), 95-129.

Borenstein, M., Hedges, L. V., Higgins, J. P. T., \& Rothstein, H. R. (2009). Introduction to meta-analysis. Chichester. Wiley.

*Bowers, P.N., y Kirby, J.R. (2010). Effects of morphological instruction on vocabulary acquisition. Reading and Writing: An Interdisciplinary Journal, 23(5), 515-537.

Carlisle, J. F. (1987). The use of morphological knowledge in spelling derived forms by learning-disabled and normal students. Annals of Dyslexia, 37, 90-108. 
Carlisle, J.F. (1996). An exploratory study of morphological errors in children's written stories. Reading and Writing, 8(1), 61-72.

Carlo, M.S., August, D., Mclaughlin, B., Snow, C.E., Dressler, Ch., Lippman, D.N., Lively, T.J. y White, C.E. (2004). Closing the gap: Addressing the vocabulary needs of English language learners in bilingual and mainstream classrooms. Reading Research Quarterly, 39(2), 188-215.

Casalis, S. y Loius-Alexandre, M. (2000). Morphological analysis, phonologi$\mathrm{cal}$ analysis and learning to read French: a longitudinal study. Reading and writing: an interdisciplinary journal, 12(3) 303-335.

*Casalis, S., y Colé, P. (2009). On the relationship between morphological and phonological awareness: Effects of training in kindergarten and in first-grade reading. First Language, 29(1), 113-142.

*Chow, B.W.-Y., Cheung, H., McBride-Chang, C. y Sze-Lok Chow, C. (2008). Dialogic reading and morphology training in Chinese children: Effects on language and literacy. Developmental Psychology, 44(1), 233-244.

Cochran, W. G. (1977) Sampling Techniques ( $3^{a}$ edición). Wiley.

Cohen J. (1988) Statistical Power Analysis for the Behavioral Sciences. Hillsdale, NJ:

Deacon, H. y Kirby, J. (2004). Morphological awareness: Just "more phonological"? The roles of morphological and phonological awareness in reading development. Applied Psycholinguistics 25, 223-238.

Deacon, S. H., y Kirby, J. R. (2004). Morphological awareness: Just «more phonological»? The roles of morphological and phonological awareness in reading development. Applied Psycholinguistics, 25(2), 223-238. doi:10.1017/S0142716404001110.

Domínguez, A., Cuetos, F. y Segui, J. (2000). Morphological processing in word recognition. Psicológica, 21, 375-401.

García, J.N. y González, L. (2006). Diferencias en la conciencia morfológica, la escritura y el lenguaje, en función del desarrollo y el nivel educativo del niño. Psicothema, 18(2), 171-179.

*Good, J. (2011). The effects of morphological awareness training on reading, spelling, and vocabulary skills. (Tesis doctoral no publicada), Philosophy department. University of Central Arkansas. Arkansas.

*Griva, E. y Anastasiou, D. (2009). Morphological strategies training the effectiveness and feasibility of morphological strategies training for students of English as a foreign language with and without spelling difficulties. Journal of Writing Research, 1(3), 199-223.

*Hamed Ghaemi, M.A. (2009). The effects of morphological training on word reading and spelling of Iranian dyslexic children. World Applied Sciencies Journal, 7(1), 57-66.

Hedges LV., Olkin, L (1985). Statistical Methods for Meta-analysis. New York, NY: Academic Press.

*Henry, M.K. (1989). Children's word structure knowledge: Implications for decoding and spelling instruction. Reading and Writing: An Interdisciplinary Journal, 1(2), 135-152.

Higgins, J.P.T. y Thompson, S.G. (2002). Quantifying heterogeneity in a meta-analysis. Statist. Med.; 21:1539-1558 (DOI: 10.1002/sim.1186).

Huedo-Medina, T.B., Sánchez-Meca, J., Marín-Martínez, F. y Botella, J. (2006). Assessing heterogeneity in meta-analysis: Q statistics or I2 index? Psychological Methods, 11(2), 193-206

Kieffer, M. J. y Lesaux, N. K. (2012a). Effects of academic language instruction on relational and syntactic aspects of morphological awareness for sixth grades from linguistically diverse backgrounds. The Elementary School Journal, 112(3), 519-545.

Kieffer, M. J. y Lesaux, N. K. (2012b) Direct and Indirect Roles of Morphological Awareness in the English Reading Comprehension of Native English, Spanish, Filipino, and Vietnamese Speakers. Language Learning, 62(4), 1170-1204.

*Kirk, C., y Gillon, G. T. (2009). Integrated Morphological Awareness Intervention as a Tool for Improving Literacy. Language, Speech, and Hearing Services in Schools, 40(3), 341-351.

Kuo, L. y. Anderson, R. (2006). Morphological Awareness and Learning to Read: A Cross-Language Perspective. Educational Psychologist, 41(3), 161180.

*Lovett, M. W. y Steinbach, K.A. (1997). The effectiveness of remedial programs for reading disabled children of different ages: does the benefit decrease for older children? Learning Disability Quarterly, 20(2), 189-209.

*Lovett, M.W., Lacerenza, L., Borden, S.L., Frijters, J.C., Steinbach, K.A. y De Palma, M. (2000). Components of effective remediation for developmental reading disabilities: combing phonological and strategy-based instruction to improve outcomes. Journal of Educational Psychology, 92(2), 263-283.

*Lyster, S.-A. H. (2002). The effects of morphological versus phonological awareness training in kindergarten on reading Effects of Instruction in Morphological Awareness on Literacy Achievement 483 development. Reading and Writing: An Interdisciplinary Journal, 15(3-4), 261-294.

McCutchen, D.; Stull, S.; Herrera, B. L.; Lotas, S.; Evans, S. (2014). Putting words to work: Effects of morphological instruction on children's writing. Journal of Learning Disabilities, 47(1), .pp.86-97.

Nagy,W., Berninger, V., Abbott, R., Vaughan, K. y Vermeulen, K. (2003). Relationship of Morphology and other language skills to literacy skills in at-risk second-grade readers and at-risk fourth-grade writers. Journal of Educational Psychology, 95(4), 730-742.

Nunes, T., Bryant, P. y Bindman, M. (2006). The effects of learning to spell on children's awareness of morphology. Reading and Writing, 19(7), $767-$ 787.

*Nunes, T., Bryant, P., y Olsson, J. (2003). Learning morphological and phonological spelling rules: An intervention study. Scientific Studies of Reading, 7(3), 289-307.

Packard, J.L., Chen, X., Li, W., Wu, X., Gaffney, J.S., Li, H, y Anderson, R.C. (2006). Explicit instruction in orthographic structure and word morphology helps Chinese children learn to write characters. Reading and Writing: An Interdisciplinary Journal, 19(5), 457-487.

*Park, Y. (2015). The roles of cognitive and language abilities of third grade students with reading disabilities responsiveness to morphological awareness intervention. Humanities and Social Sciences, 75(7-A).

Perfetti, C. (2007). Reading ability: Lexical quality to comprehension. Scientific Studies of Reading, 11(4), 357-383. doi:10.1080/10888430701530730.

Ramírez, G., Chen, X., Geva, E. y Kiefer, H. (2010). Morphological awareness in Spanish-speaking English language learners: Within and crosslanguage effects on word reading. Reading and Writing, 23 (3-4), 337-358.

Raudenbush, S. W. (2009). Analyzing effect sizes: random-effects models. En H. Cooper, L. V. Hedges, y J. C. Valentine (Eds.), The handbook of research synthesis and meta-analysis (pp. 295-314). Nueva York: Russell Sage.

*Rosa, J.M. y Nunes, T. (2008). Morphological priming effects on children's spelling. Reading and Writing, 21(8), 763-781.

Rubin, H. (1991). Morphological knowledge and writing ability. En J.R. Malatesha (Ed). Written language disorders (pp.43-69). Nueva York, NY: Kluwer Academic/Plenum Publishers.

Rueda, M. I. e Incera, S. (2011). Diferencias en conciencia morfológica entre buenos lectores y alumnos con dificultades en lectoescritura. Capítulo de libro Educación Aprendizaje y desarrollo en una sociedad Multicultural (pp. 8327-8339). En J. M. Román Sánchez, M. A. Carbonero Martín y J. D. Valdivieso Pastor (compiladores). Madrid: Ediciones de la Asociación Nacional de Psicología y Educación.

*St-Pierre, M-C. y Dubé, J-F. (2012). Morphological awareness intervention: does severity play a role in spelling/reading improvement? Aula Abierta, 40(3), 15-22.

Tong, X., Deacon, S.H., Kirby, J.R, Cain, K. y Parrilla, R. (2011). Morphological Awareness: A Key to Understanding Poor Reading Comprehension in English. Journal of Educational Psychology, 103(3), 523-534.

*Tsesmeli, S.N. y Seymour, P.H. (2009). The effects of training of morphological structure on spelling derived words by dyslexic adolescents. British Journal of Psychology, 100(3), 565-592.

*Vadasy, P. F., Sanders, E. A. y Peyton, J. A. (2006). Paraeducator supplemented instruction in structural analysis with text reading practice for second and third graders at risk for reading problems. Remedial and Special Education, 27, 365-378.

*Wu, X., Anderson, R.C., Li, W., Wu, X., Li, H., Zhang, J., Zheng, Q., Zhu, J., Shu, H., Jiang, W., Chen, X., Wang, Q., Yin, L., He, Y., Packard, J. y Gaffney, J.S. (2009). Morphological awareness and Chinese children's literacy development: An intervention study. Scientific Studies of Reading, 13(1), 26-52.

(* Estudios incluidos en el meta-análisis)

(Artículo recibido: 03-04-2014; revisado: 24-11-2014; aceptado: 09-05-2015) 